\title{
Clinical Study Bronchial Responsiveness in Patients with
Restrictive Spirometry
}

\author{
Jean I. Keddissi, Marwan K. Elya, Saif U. Farooq, Houssein A. Youness, \\ Kellie R. Jones, Ahmed Awab, and Gary T. Kinasewitz
}

Division of Pulmonary/Critical Care Medicine, the Oklahoma City VA Medical Center and the University of Oklahoma Health Sciences Center, 920 Stanton L. Young Boulevard, WP 1310, Oklahoma City, OK 73104-5020, USA

Correspondence should be addressed to Jean I. Keddissi; jean-keddissi@ouhsc.edu

Received 4 April 2013; Accepted 11 July 2013

Academic Editor: Demosthenes Bouros

Copyright (C) 2013 Jean I. Keddissi et al. This is an open access article distributed under the Creative Commons Attribution License, which permits unrestricted use, distribution, and reproduction in any medium, provided the original work is properly cited.

Background. Improvement in PFT after bronchodilators is characteristic of obstructive airway diseases such as COPD. However, improvement in patients with restrictive pattern is occasionally seen. We aim to determine the clinical significance of a bronchodilator responsive restrictive defect. Methods. Patients with restrictive spirometry and a bronchodilator study were identified at the University of Oklahoma and Oklahoma City VAMC between September 2003 and December 2009. Restriction was defined as a decreased FVC and FEV1, with normal FEV1/FVC. Responsiveness to bronchodilators was defined as an improvement in FEV1 and/or FVC of at least 12\% and $200 \mathrm{~mL}$. Patients with lung volume measurements had their clinical and radiographic records reviewed. Results. Twenty-one patients were included in the study. Most were current or ex-smokers, with most being on bronchodilators. The average FVC and FEV1 were $65 \pm 11 \%$ and $62 \pm 10 \%$ of the predicted, respectively. Most patients (66\%) had a normal TLC, averaging $90 \pm 16 \%$ of the predicted. RV, RV/TLC, and the TLC-VA values strongly suggested an obstructive defect. Conclusions. Reversible restrictive pattern on spirometry appears to be a variant of obstructive lung disease in which early airway closure results in air trapping and low FVC. In symptomatic patients, a therapeutic trial of bronchodilators may be beneficial.

\section{Introduction}

The use of short-acting bronchodilators, such as albuterol, during pulmonary function tests (PFTs) is a very common, routine test. Bronchial responsiveness to bronchodilators is a physiological response involving airway epithelium, nerves, mediators, and bronchial smooth muscles [1]. Based on the ATS guidelines, such responsiveness is considered to be present when the improvement in forced expiratory volume in 1 second (FEV1) or in the forced vital capacity (FVC) is at least $12 \%$ and $200 \mathrm{~mL}$ [1]. Increments of less than $8 \%$ are likely to be within measurement variability.

The test has diagnostic, therapeutic, and prognostic implications in patients with obstructive disease $[2,3]$, such as asthma and chronic obstructive pulmonary disease (COPD). Its significance, when positive in a nonobstructive case (i.e., normal or restrictive spirometry) remains to be determined.

While several case reports have described individual patients with a reversible restrictive lung patterns [4-8], the significance of a positive bronchial responsiveness test in a group of patients with purely restrictive spirometry has not been examined. Our aim was to evaluate the clinical characteristics of patients with restrictive spirometry and a significant response to bronchodilators and to distinguish between restrictive and obstructive disease based on their radiographic and lung volume findings. Our hypothesis is that bronchodilator responsiveness in patients with restrictive defect is likely indicative of an underlying obstructive airway disease.

\section{Methods}

This was a retrospective cohort study conducted at the University of Oklahoma and the Oklahoma City Veterans Affairs Medical Center. None of the authors has any conflict of interest associated with the study. After obtaining IRB approval to conduct the study (IRB number 13098), all PFTs meeting ATS criteria for acceptability and reproducibility 
[9], performed between September 2003 and December 2009 on patients older than 18, were reviewed. All patients with an initial restrictive spirometry, with significant response to bronchodilators who had pulmonary lung volumes measured, were included in our analysis.

All the studies were done by experienced pulmonary technologists, using SensorMedics Vmax 229D series (CareFusion, San Diego, CA, USA). Lung volumes were done on a V6200 AutoBox (CareFusion, San Diego, CA, USA). Normal spirometry values were calculated from the NHANES III database [10], while lung volumes and diffusion capacity (DLCO) normal values were derived from the studies by Crapo et al. $[11,12]$. We defined a restrictive spirometry as one with a FVC <lower limit of normal (i.e., 5 th percentile), with a normal FEV1/FVC. Normal FEV1/FVC was defined based on the NHANES III database and the ATS guidelines as values greater than $95 \%$ of the predicted for age, gender, and height $[1,10]$. Positive response to bronchodilators was defined as a change in FEV1 or in the FVC of at least $12 \%$ and $200 \mathrm{~mL}$ [1]. Prebronchodilator maximum voluntary ventilation (MVV) was recorded, was considered decreased if it fell below the lower limit of normal, and was $<32 x F E V 1$ [9]. Lung volumes were measured using whole-body plethysmography. DLCO and alveolar volume (VA) were obtained by the single-breath method. Airway resistance (Raw) and specific conductance (sGaw) were measured and compared to reference values [13].

Medical records were reviewed. Patients characteristics, including demographics, smoking history, comorbidities, medication use, and symptoms at the time of the initial spirometry, were recorded. Radiographic findings on chest Xrays were also included. Data is expressed as mean \pm standard deviation (SD). The percentage of patients with a decreased TLC (suggestive of a true restrictive defect) was calculated.

\section{Results}

Between September 2003 and December 2009, there were 7502 spirometries with bronchodilator evaluations performed and 1588 plethysmographic lung volume measurements, all done using whole-body plethysmography. During that period, we identified 21 patients with a reversible restrictive pattern on PFT, who had lung volumes measured. The average age was 65 years (range 48 to 81 ) (Table 1). Fifteen patients were male (71\%), while the majority was Caucasian (86\%). Only 6 patients (29\%) never smoked. In those with a history of smoking, the average pack-year was $51 \pm 29$. The patients tended to be overweight, with a body mass index (BMI) of $31 \pm 7 \mathrm{~kg} / \mathrm{m}^{2}$ (range $23-47 \mathrm{~kg} / \mathrm{m}^{2}$ ).

Most patients were symptomatic (Table 2). Dyspnea was the main reason to obtain the PFT, followed by cough and wheezing. Only 1 patient did not have any of these complaints. Most patients did not show radiographic findings suggestive of a restrictive disease or of a chronic obstructive disease. More than half had a clinical history suggestive of asthma or COPD. One patient had a history of interstitial lung disease, while another two patients had a history of autoimmune disease without evidence of pulmonary involvement. The majority of patients were on inhaled bronchodilators and/or inhaled corticosteroids.
TABLE 1: Demographics of the 21 patients with reversible restrictive spirometry.

\begin{tabular}{lc}
\hline Age $(\text { years })^{*}$ & $65 \pm 9$ \\
Gender $(\mathrm{M} / \mathrm{F})$ & $15 / 6$ \\
Race & \\
$\quad$ Caucasian & $18(86 \%)$ \\
$\quad$ African-American & $3(14 \%)$ \\
Smokers $(n, \%)$ & \\
$\quad$ Current & $7(33 \%)$ \\
$\quad$ Ex-smoker & $8(38 \%)$ \\
$\quad$ Never & $6(29 \%)$ \\
Pack-year & $51 \pm 29$ \\
Body mass index $\left(\mathrm{kg} / \mathrm{m}^{2}\right)^{*}$ & $31 \pm 7$ \\
\hline
\end{tabular}

${ }^{*}$ Data expressed as mean $\pm \mathrm{SD}$.

TABLE 2: Symptoms, radiographic findings, and medical history.

\begin{tabular}{lc}
\hline & $n(\%)$ \\
\hline Symptoms & $13(62 \%)$ \\
Cough & $18(86 \%)$ \\
Dyspnea & $7(39 \%)$ \\
Wheezing $^{*}$ & \\
Radiographic findings $^{\S}$ & $2(10 \%)$ \\
Fibrotic changes & $4(20 \%)$ \\
COPD changes & $4(20 \%)$ \\
Kyphosis/scoliosis & \\
Past medical history & \\
Asthma & $6(32 \%)$ \\
COPD & $4(21 \%)$ \\
Interstitial lung disease & $1(5 \%)$ \\
Chronic inflammatory/autoimmune disease & $2(11 \%)$ \\
Medications & \\
Bronchodilators & $14(74 \%)$ \\
Inhaled corticosteroids & $10(53 \%)$ \\
Systemic steroids & $2(11 \%)$ \\
Home O & $3(16 \%)$ \\
\hline
\end{tabular}

${ }^{*}$ Wheezing data not available in $3 / 21$ patients. ${ }^{\S} \mathrm{CXR}$ data not available in $1 / 21$ patient. ${ }^{¥}$ Medication data not available in $2 / 21$ patients.

Table 3 summarizes the baseline spirometry data. All patients had a restrictive defect on spirometry, with an average FEV1 of $62 \pm 10 \%$ and FVC of $65 \pm 11 \%$. FEV1/FVC and FEV1/SVC were all normal. All patients had a significant response to bronchodilators (either by FEV1 or FVC criteria), with 10 patients having response to both. The MVV was reduced in 9 patients (43\%).

The total lung capacity (TLC) averaged $5.78 \pm 1.44 \mathrm{~L}$ or $90 \pm 16 \%$ of the predicted and was below the lower limit of normal in 7 patients (33\%) (Table 4). In those 7 cases, the average TLC was $74 \%$ of the predicted. In 2 patients, the TLC was above the upper limit of normal. The average residual volume (RV) was high at $128 \pm 38 \%$. No RV fell below the lower limit of normal. The RV/TLC was elevated with a mean of $52 \pm 9 \%$. Airway resistance (Raw) was elevated in 16 patients 
TABLE 3: Baseline pulmonary function tests.

\begin{tabular}{lc}
\hline FEV1 & \\
Observed, (L) & $1.91 \pm 0.58$ \\
$\%$ predicted & $62 \pm 10$ \\
<Lower limit of normal & $20(95)$ \\
Bronchodilator response (L) & $0.32 \pm 0.15$ \\
Bronchodilator response (\%) & $17.1 \pm 6.3$ \\
FVC & \\
Observed, (L) & $2.62 \pm 0.82$ \\
\% predicted & $65 \pm 11$ \\
<Lower limit of normal & $21(100)$ \\
Bronchodilator response (L) & $0.41 \pm 0.15$ \\
Bronchodilator response (\%) & $16.5 \pm 6.1$ \\
FEV1/FVC & $73 \pm 5$ \\
FEV1/SVC & $72 \pm 5$ \\
MVV & \\
Observed, (L) & $63 \pm 23$ \\
\% predicted & $52 \pm 16$ \\
$<$ Lower limit of normal and $<32 \times$ FEV1 & $9(43)$ \\
\hline
\end{tabular}

Prebronchodilators values are presented as mean \pm SD or $n(\%)$.

TABLE 4: Lung volume measurements.

\begin{tabular}{|c|c|}
\hline TLC & \\
\hline Observed, (L) & $5.78 \pm 1.44$ \\
\hline$\%$ predicted & $90 \pm 16$ \\
\hline$<$ Lower limit of normal & $7(33)$ \\
\hline TLC-VA (L) & $1.27 \pm 0.90$ \\
\hline $\mathrm{VC}$ & \\
\hline Observed, (L) & $2.75 \pm 0.83$ \\
\hline$\%$ predicted & $68 \pm 13$ \\
\hline$<$ Lower limit of normal & $18(86)$ \\
\hline RV & \\
\hline Observed, (L) & $3.03 \pm 0.96$ \\
\hline$\%$ predicted & $128 \pm 38$ \\
\hline$<$ Lower limit of normal & $0(0)$ \\
\hline $\mathrm{RV} / \mathrm{TLC}$ & \\
\hline Observed & $0.52 \pm 0.09$ \\
\hline$>$ Upper limit of normal & $10(48 \%)$ \\
\hline Adjusted DLCO & \\
\hline Observed, $(\mathrm{mL} / \mathrm{mmHg} / \mathrm{min})$ & $17.37 \pm 6.97$ \\
\hline$\%$ predicted & $69 \pm 24$ \\
\hline$<$ Lower limit of normal & $12(57)$ \\
\hline Raw & \\
\hline$\%$ predicted & $253 \pm 150$ \\
\hline >Upper limit of normal & $16(76 \%)$ \\
\hline sGaw & \\
\hline$\%$ predicted & $42 \pm 21$ \\
\hline
\end{tabular}

Values are presented as mean \pm SD or $n$ (\%). Raw: airway resistance; sGaw: specific conductance.

(76\%) with a mean of $253 \pm 150 \%$, while the average specific conductance was reduced at $42 \pm 21 \%$.

The mean adjusted DLCO was $69 \pm 24 \%$. In 12 patients (57\%), the DLCO was below the lower limit of normal. The average TLC-VA difference was $1.27 \pm 0.90 \mathrm{~L}$ (range 0.33$3.54 \mathrm{~L})$.

\section{Discussion}

Pulmonary function testing is an invaluable tool in the hands of pulmonologists and primary care physicians. It is used primarily as a diagnostic aide during the workup of respiratory symptoms, but PFTs are also helpful in the long-term patient monitoring as well as evaluation of potential disability and in epidemiological and research studies [9]. One of the first questions spirometry has to answer is whether an obstructive abnormality (defined as a reduced FEV1/VC below the 5th percentile of the predicted value) or a restrictive one (defined as reduced VC with normal or slightly increased FEV1/VC) is present [1]. Since the vital capacity may be diminished by both restrictive and obstructive ventilatory defects [14], a reduced $\mathrm{VC}$ does not prove the presence of a restrictive defects, and the presence of which has to be confirmed by low TLC. In fact, Aaron et al. showed that $<60 \%$ of patients with a spirometric restrictive pattern (i.e., low FVC and normal or above normal FEV1/FVC) had pulmonary restriction confirmed on lung volume measurements [15]. Failure to exhale long enough to empty the lung to RV can result in a decreased FVC and FEV1 with a normal FEV1/FVC [1]. In addition, early airway closure with gas trapping may lead to airflow limitation at low lung volume, with incomplete lung emptying and hyperinflation [14]. This could be interpreted as a restrictive defect, when the true underlying pathology is an obstructive one. Slow VC may give a better estimate of the vital capacity, but measurement of lung volumes is usually needed to exclude restriction (normal TLC) and to show evidence of air trapping, as manifested by an increased RV and RV/TLC.

A mixed pattern is defined as a FEV1/VC and a TLC that are both below the 5th percentiles of their predicted values.

Bronchodilators during spirometry are typically used to assess the response of an obstructive defect. It is well recognized that the response could be seen in the FEV1, FVC, or both. Even though there is no consensus about what constitutes reversibility in patients with obstruction [16], the American Thoracic Society/European Respiratory Society defines significant response as $>12 \%$ and $200 \mathrm{~mL}$ compared with baseline [1].

Our study is the first to systematically examine the clinical significance of a restrictive pattern (defined as a spirometry with decreased FVC, FEV1 with normal FEV1/FVC) that is responsive to bronchodilators in a group of patients referred for pulmonary function testing. The slow VC was measured at baseline in 13 patients. In all these cases the FEV1/SVC was within normal limits and therefore could not be used to infer the presence of an obstructive defect. Despite a spirometry that would be interpreted as showing moderate restriction, the TLC was normal in two-thirds of our patients, excluding significant restriction in these subjects [17]. None of our patients had a decreased RV as might be expected in a restrictive defect. In fact, the average RV/TLC was elevated at $52 \pm 9 \%$, with 10 patients $(48 \%)$ having a ratio that was $>95 \%$ of the predicted value for their age [18]. This would be expected to result from air trapping, a hallmark of obstructive lung diseases. In fact, most of our patients were current or exsmokers, which puts them at risk for obstructive lung disease. In addition, the large difference between the TLC and alveolar 
volume (TLC-VA) points in the same direction and suggests maldistribution of ventilation as the result of an obstructive dysfunction. The elevated airway resistance with a reduced specific conductance is another typical finding of obstructive lung disease. Finally the measured DLCO was reduced in the majority of patients. Even though this is a feature of restrictive lung disease, it is also seen in obstructive pulmonary disease, such as emphysema [19].

Previous case reports have described individual patients with a reversible or bronchodilator responsive restrictive lung disease manifest by both a restrictive spirometric pattern and a reduced TLC. This reduction in lung volume has been attributed to the closure of the terminal lung units by contraction of alveolar ducts [5]. Miller and Palecki who reviewed the PFTs and medical records of 413 patients with a clinical diagnosis of asthma found that a restrictive pattern was relatively common and usually associated with an elevated BMI [20]. Pseudorestriction with air trapping was seen in only 7 patients. This is in contrast to the present study in which half of the patients had an elevated residual volume.

The majority of patients in our study displayed the "nonspecific pattern" of pulmonary function test, in which FEV1 and FVC are reduced, while the FEV1/FVC and TLC are normal, described by Hyatt et al. [21]. This pattern was seen in approximately $10 \%$ of patients undergoing spirometry and lung volume measurements at the Mayo Clinic. A similar group of 12 patients was described in 2004 and were thought to suffer from small airway obstruction [22]. In a larger follow-up study from the Mayo Clinic group, a significant response to bronchodilators was seen in $11 \%$ of the patient with the nonspecific pattern [23]. While a response to bronchodilators, history of smoking, and large TLC-VA difference predicted the development of an obstructive defect, most patients continue to exhibit the same pattern on subsequent spirometry. This "small airways obstruction syndrome" is thought to be present in early emphysema, small airway disease, asthma, and in older age [24]. Even though a few our patients had a low TLC, the majority of our population are likely to be a different manifestation of the same phenomenon, where an obstructive defect does not present with the classical reduction of the FEV1/FVC. Rather, the clue to the presence of the obstructive defect is the response to bronchodilators.

Due to the retrospective nature of our study, we cannot eliminate a selection or history bias. Lung volume measurements were not uniformly done in all patients with restrictive spirometry and bronchodilator response. Therefore, our group may not be truly representative of this condition. In addition, we cannot have a definite estimate of its true incidence. We only identified 21 patients who met these criteria, out of a total of 7502 studies suggesting that this is a rather rare event. However, since our group may have similar characteristics to the Mayo Clinic subgroup of patients with nonspecific pattern who were responsive to bronchodilators $[21,23]$, our best estimate from these 2 studies is that $1 \%$ of patients undergoing spirometry and lung volume may end up having this condition ( $11 \%$ response to bronchodilator among the $10 \%$ of patients in whom the FEV1 and FVC are reduced, while the FEV1/FVC and TLC are normal). Prospectively designed studies where all patients with this condition have measurements of their lung volumes will be needed to address these limitations.

In summary, our report is the first to exclusively describe patients with a restrictive spirometry that is responsive to bronchodilators. In most cases, lung volume measurements exclude a significant restrictive defect and suggest the presence of an obstructive one. This is likely due to early airway closure resulting in air trapping. Clinicians need to be aware of this phenomena, and the possibility of underlying obstructive diseases needs to be strongly considered. Symptomatic patients may benefit from a therapeutic trial of bronchodilators.

\section{Conflict of Interests}

The authors declared that they have no conflict of interests.

\section{Acknowledgment}

Preliminary data was presented in an abstract form at the annual meetings of the American College of Chest Physicians in October 2007 and October 2008.

\section{References}

[1] R. Pellegrino, G. Viegi, V. Brusasco et al., "Interpretative strategies for lung function tests," European Respiratory Journal, vol. 26, no. 5, pp. 948-968, 2005.

[2] G. H. Guyatt, M. Townsend, S. Nogradi, S. O. Pugsley, J. L. Keller, and M. T. Newhouse, "Acute response to bronchodilator. An imperfect guide for bronchodilator therapy in chronic airflow limitation," Archives of Internal Medicine, vol. 148, no. 9, pp. 1949-1952, 1988.

[3] P. L. P. Brand, P. H. Quanjer, D. S. Postma et al., "Interpretation of bronchodilator response in patients with obstructive airways disease," Thorax, vol. 47, no. 6, pp. 429-436, 1992.

[4] R. Gilbert and J. H. Auchincloss Jr., "Reactive airways dysfunction syndrome presenting as a reversible restrictive defect," Lung, vol. 167, no. 1, pp. 55-61, 1989.

[5] D. A. Kaminsky and C. G. Irvin, "Anatomic correlates of reversible restrictive lung disease," Chest, vol. 103, no. 3, pp. 928-931, 1993.

[6] D. W. Hudgel, D. Cooper, and J. Souhrada, "Reversible restrictive lung disease simulating asthma," Annals of Internal Medicine, vol. 85, no. 3, pp. 328-332, 1976.

[7] C. Colp and M. H. Williams Jr., "Total occlusion of airways producing a restrictive pattern of ventilatory impairment," The American Review of Respiratory Disease, vol. 108, no. 1, pp. 118122, 1973.

[8] D. P. Schlueter, J. N. Fink, and A. J. Sosman, "Pulmonary function in pigeon breeders' disease: a hypersensitivity pneumonitis," Annals of Internal Medicine, vol. 70, no. 3, pp. 457-470, 1969.

[9] M. R. Miller, J. Hankinson, V. Brusasco et al., "Standardisation of spirometry," European Respiratory Journal, vol. 26, no. 2, pp. 319-338, 2005.

[10] J. L. Hankinson, J. R. Odencrantz, and K. B. Fedan, "Spirometric reference values from a sample of the general U.S. Population," The American Journal of Respiratory and Critical Care Medicine, vol. 159, no. 1, pp. 179-187, 1999. 
[11] R. O. Crapo, A. H. Morris, P. D. Clayton, and C. R. Nixon, "Lung volumes in healthy nonsmoking adults," Clinical Respiratory Physiology, vol. 18, no. 3, pp. 419-425, 1982.

[12] R. O. Crapo and A. H. Morris, "Standardized single breath normal values for carbon monoxide diffusing capacity," The American Review of Respiratory Disease, vol. 123, no. 2, pp. 185-189, 1981.

[13] A. B. Dubois, S. Y. Botelho, and J. H. Comroe Jr., "A new method for measuring airway resistance in man using a body," Journal of Clinical Investigation, vol. 35, no. 3, pp. 327-335, 1956.

[14] P. H. Quanjer, G. J. Tammeling, J. E. Cotes, O. F. Pedersen, R. Peslin, and J. C. Yernault, "Lung volumes and forced ventilatory flows. Report Working Party Standardization of Lung Function Tests, European Community for Steel and Coal. Official Statement of the European Respiratory Society," The European Respiratory Journal, vol. 16, supplement, pp. 5-40, 1993.

[15] S. D. Aaron, R. E. Dales, and P. Cardinal, "How accurate is spirometry at predicting restrictive pulmonary impairment?" Chest, vol. 115, no. 3, pp. 869-873, 1999.

[16] O. Eliasson and A. C. Degraff Jr., "The use of criteria for reversibility and obstruction to define patient groups for bronchodilator trials. Influence of clinical diagnosis, spirometric, and anthropometric variables," The American Review of Respiratory Disease, vol. 132, no. 4, pp. 858-864, 1985.

[17] R. E. Hyatt, P. D. Scanlon, and M. Nakamura, Interpretation of Pulmonary Function Tests, Lippincott-Raven, New York, NY, USA, 1997.

[18] J. Stocks and P. H. Quanjer, "Reference values for residual volume, functional residual capacity and total lung capacity: ATS Workshop on Lung Volume Measurements Official Statement of the European Respiratory Society," European Respiratory Journal, vol. 8, no. 3, pp. 492-506, 1995.

[19] N. J. Morrison, R. T. Abboud, F. Ramadan et al., "Comparison of single breath carbon monoxide diffusing capacity and pressurevolume curves in detecting emphysema," The American Review of Respiratory Disease, vol. 139, no. 5, pp. 1179-1187, 1989.

[20] A. Miller and A. Palecki, "Restrictive impairment in patients with asthma," Respiratory Medicine, vol. 101, no. 2, pp. 272-276, 2007.

[21] R. E. Hyatt, C. T. Cowl, J. A. Bjoraker, and P. D. Scanlon, "Conditions associated with an abnormal nonspecific pattern of pulmonary function tests," Chest, vol. 135, no. 2, pp. 419-424, 2009.

[22] D. Stănescu and C. Veriter, "A normal FEV1/VC ratio does not exclude airway obstruction," Respiration, vol. 71, no. 4, pp. 348352, 2004.

[23] V. N. Iyer, D. R. Schroeder, K. O. Parker, R. E. Hyatt, and P. D. Scanlon, "The nonspecific pulmonary function test: longitudinal follow-up and outcomes," Chest, vol. 139, no. 4, pp. 878-886, 2011.

[24] D. Stǎnescu, "Small airways obstruction syndrome," Chest, vol. 116, no. 1, pp. 231-233, 1999. 


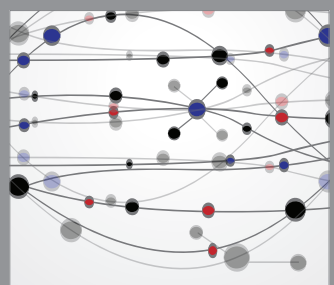

The Scientific World Journal
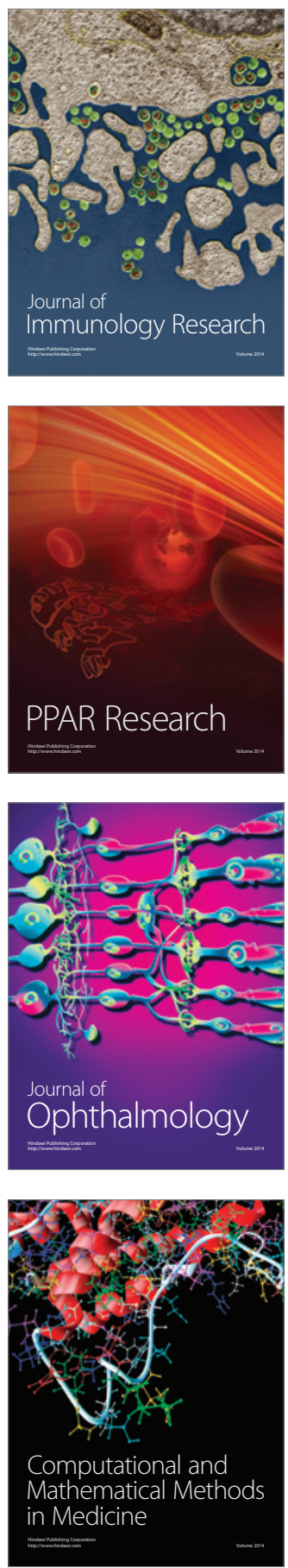

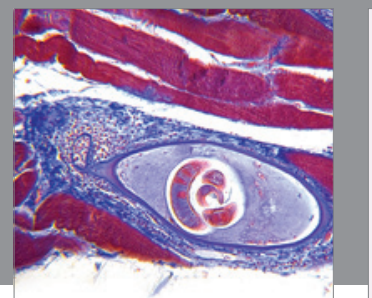

Gastroenterology

Research and Practice
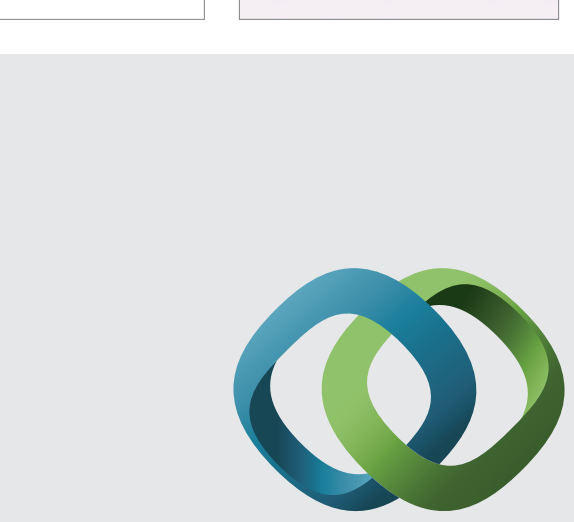

\section{Hindawi}

Submit your manuscripts at

http://www.hindawi.com
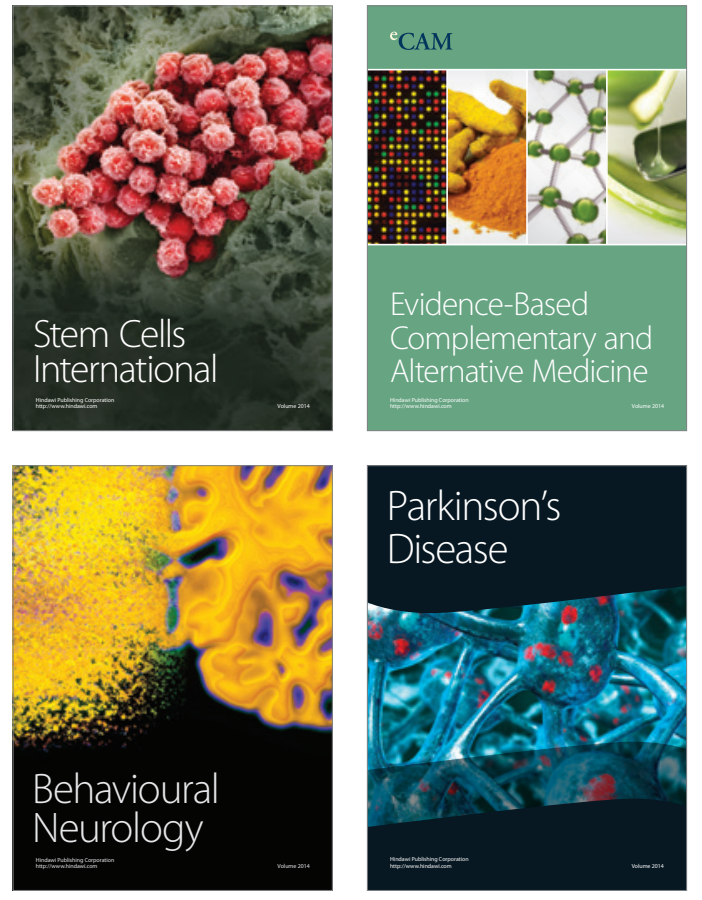
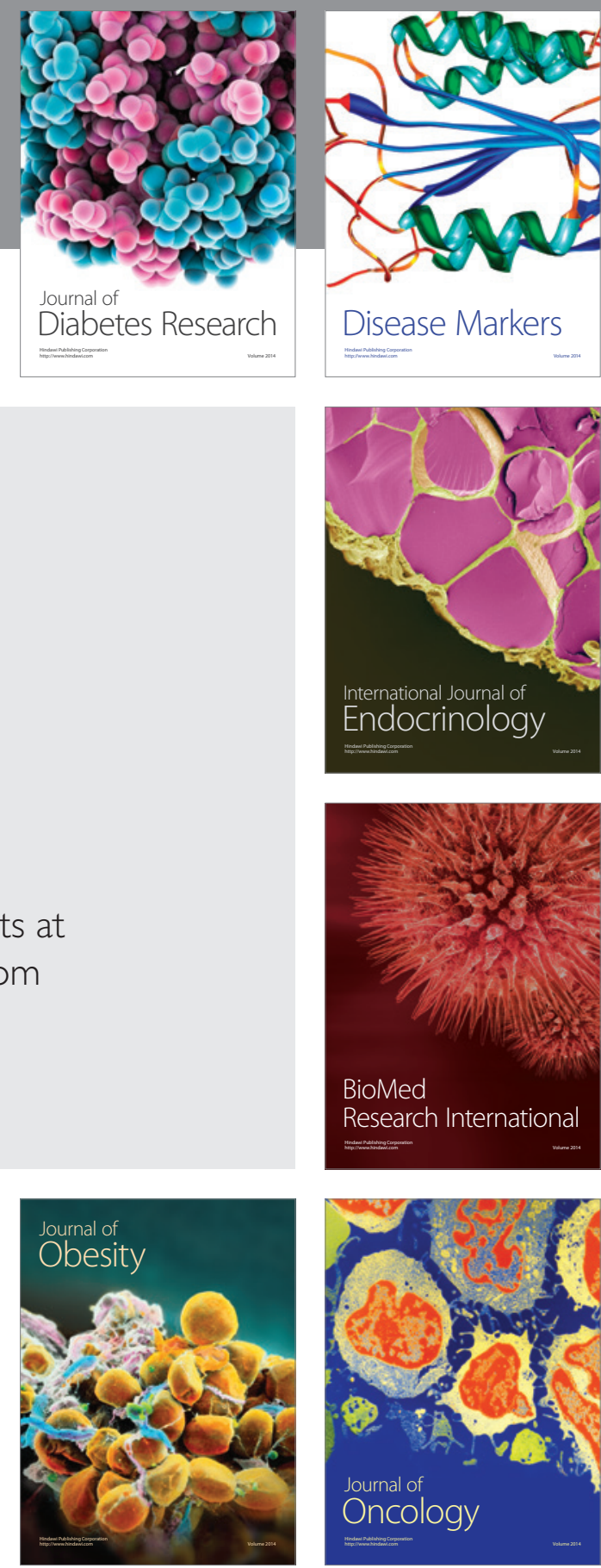

Disease Markers
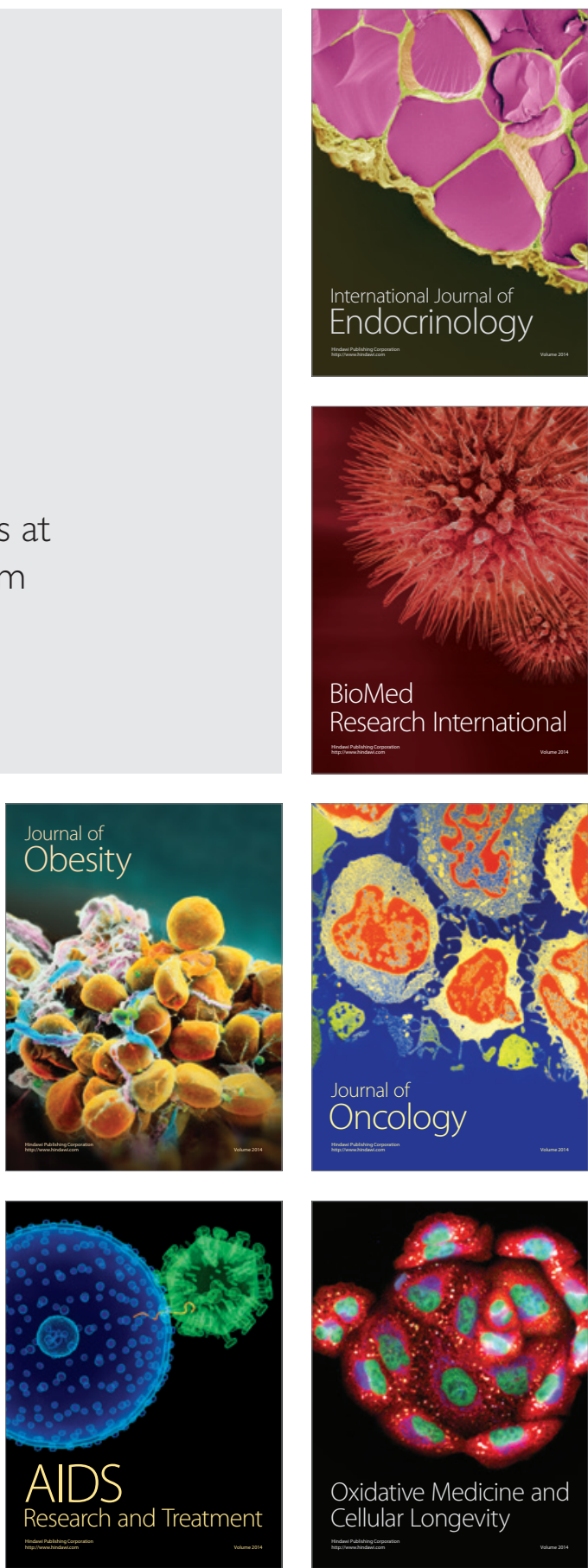